\title{
Infinite horizon LQ optimal output tracking from development to real flight tests
}

\author{
Péter Bauer Computer and Automation Research Institute \\ Hungarian Academy of Sciences \\ Budapest, Hungary \\ Email: bauer.peter@sztaki.hu József Bokor Computer and Automation Research Institute \\ Hungarian Academy of Sciences \\ Budapest, Hungary
}

\begin{abstract}
This paper introduces a new LQ optimal, infinite horizon output tracking solution. It publishes results from problem formulation to real flight tests. After a short literature review, it rigorously derives the problem solution and states all of the properties of this new control method. Then it introduces the lateral-directional aircraft model on which the algorithm was tuned and tested. The LQ optimal algorithm was compared by a baseline PID control solution in several simulations and real flight tests. Finally, the pros and cons are summarized and the future direction of development is mentioned.
\end{abstract}

\section{INTRODUCTION}

LQ optimal state and output tracking control is an actively researched field since the 1960s. Several exact or approximate solutions were derived using different approaches. The existing methods can be classified in two main groups, such as a priori given reference signals over the known future horizon and solutions using only instantaneous and past reference values. The authors are interested in the latter techniques which provide real time applicability.

LQ Servo (integral) control in [1] requires the implementation of additional integrators which increases system dimension. These new states are related to the output error terms.

An alternate technique in [2] deals with continuous time (CT) output tracking, considering linear time varying (LTV) finite horizon and linear time invariant (LTI) large horizon solutions. The finite horizon solution is rigorously derived and contains state feedback with an extra forcing function. Both the LTV and the LTI optimal solutions have to be calculated backward in time, so these approaches need the reference signal in advance. Nevertheless, the large horizon solution is only an approximation, it does not consider infinite time. The solution assumes to have a constant reference signal. A discrete time representation is derived for the same problems in [3] and [4].

The large horizon technique proposed in [2] and [3] is further improved in [5] and [6].

In [5], the CT infinite horizon problem for constant reference tracking has been elaborated but asymptotical tracking can not be guaranteed with.

In [6], the authors derive a system of algebraic equations based on the initial state of the forcing function (see also [2]).
The backward recursion is avoided, but the structure of the reference signal has to be fixed (assumed to be polynomial).

The author's previous works ([7] and [8]) deal with the problem of deriving an infinite horizon LQ optimal output tracking solution which guarantees zero steady state tracking error for constant references. Unfortunately the derived solution is not optimal, because finite functional value can not be guaranteed even for constant references.

In this paper the results of the previous papers are revised and an optimal (for constant references) and sub-optimal (for time-varying references) solution is derived in a unified framework. The derived method is applied in the roll angle reference tracking control of an aircraft. It was tested and compared by a baseline PID solution ([9]) in Matlab simulations and real flights.

The outline of the paper is as follows: Section II deals with the problem formulation and solution. Section III introduces the lateral dynamical model of the aircraft on which the method was tuned and tested and describes the tuning and testing procedure. Section IV publishes the simulation and flight test results and finally, the paper ends with the conclusion.

\section{PROBlem FORMUlation AND SOLUtion}

This article deals with discrete time, linear, time invariant systems in the following form:

$$
\begin{aligned}
x_{k+1} & =A x_{k}+B \tilde{u}_{k}+W w_{k} \\
y_{k}^{r} & =C_{r} x_{k} \\
y_{k} & =C x_{k}+V v_{k}
\end{aligned}
$$

Where $x_{k} \in \mathbb{R}^{n}, \quad \tilde{u}_{k} \in \mathbb{R}^{m}, \quad y_{k}^{r} \in \mathbb{R}^{r}, \quad y_{k} \in \mathbb{R}^{p}, \quad w_{k} \in$ $\mathbb{R}^{w}, \quad v_{k} \in \mathbb{R}^{v}$ are the system state, input, tracking output, measured output, stochastic disturbance and measurement noise respectively and the matrices $A, B, C_{r}, C, W, V$ have appropriate dimensions. It is assumed that the pair $(A, B)$ is stabilizable and the pair $(C, A)$ is observable. Such system model can well describe the motion of an aircraft around a trim point subject to stochastic wind disturbances for example.

The goal is to track constant or time-varying references with the tracking output. It is assumed that the noise effects are handled by the state estimator. The derived solution is a multi step algorithm as follows: 
1) Design a stabilizing state feedback controller for the pair $(A, B)$ if $\mathrm{A}$ is unstable (either with pole placement or LQ optimal design) in the form: $\tilde{u}_{k}=-K_{x 1} x_{k}+u_{k}$

2) Determine the solution of the steady state constant reference tracking problem considering the stabilized system with $\phi=A-B K_{x 1}$

$$
\begin{aligned}
& x_{\infty}=\phi x_{\infty}+B u_{\infty} \\
& y_{\infty}=C_{r} x_{\infty}=r_{\infty} \\
& \underbrace{C_{r}(I-\phi)^{-1} B}_{F} u_{\infty}=r_{\infty} \\
& u_{\infty}=\operatorname{pinv}(F) r_{\infty}
\end{aligned}
$$

3) Construct an LQ sub-optimal tracking controller for time varying references, centering the original system with the setady state and applying linear extrapolation. The result will be LQ optimal for constant references. The centered state equations:

$$
\begin{aligned}
& x_{k+1}-x_{\infty}=\phi\left(x_{k}-x_{\infty}\right)+B\left(u_{k}-u_{\infty}\right) \\
& \Delta x_{k+1}=\phi \Delta x_{k}+B \Delta u_{k} \\
& \Delta y_{k}=C_{r} \Delta x_{k} \\
& \Delta r_{k}=r_{k}-r_{\infty}
\end{aligned}
$$

The functional to be minimised:

$$
\begin{aligned}
& J(\Delta x, \Delta \tilde{x}, \Delta u)= \\
& =\frac{1}{2} \sum_{k=0}^{\infty}\left[\left(\Delta x_{k}-\Delta \tilde{x}_{k}\right)^{T} Q\left(\Delta x_{k}-\Delta \tilde{x}_{k}\right)+\right. \\
& \left.+\Delta u_{k}^{T} R \Delta u_{k}\right] \\
& \text { where }: \\
& \Delta \tilde{x}_{k}=C_{r}^{T}\left(C_{r} C_{r}^{T}\right)^{-1} \Delta r_{k}=H \Delta r_{k} \\
& \bar{C}=I-C_{r}^{T}\left(C_{r} C_{r}^{T}\right)^{-1} C_{r} \\
& Q=\bar{C}^{T} Q_{1} \bar{C}+C_{r}^{T} Q_{2} C_{r}
\end{aligned}
$$

Here, $Q_{2}$ weights the tracking error, while $Q_{1}$ weights the states not included in the tracking output.

4) Sum up all the control input components from steps 1-3.

The solution in the 3rd step was derived using Lagrange multiplier method. The costate variable resulted as (parameterized by $P \infty, S_{1}$ and $S_{2}$ ):

$$
\begin{aligned}
& \lambda_{k+1}=P_{\infty}\left[I+B R^{-1} B^{T} P_{\infty}\right]^{-1} \phi \Delta x_{k}- \\
& -\left[I+P_{\infty} B R^{-1} B^{T}\right]^{-1} S_{1} \Delta r_{k+1}+ \\
& +\left[I+P_{\infty} B R^{-1} B^{T}\right]^{-1} S_{2} \Delta r_{k+2}
\end{aligned}
$$

from which the following system of equations results considering the condition for optimality: $\lambda_{k}=Q \Delta x_{k}-Q H \Delta r_{k}+$ $\phi^{T} \lambda_{k+1}$ and introducing $M_{2}=\left[I+P_{\infty} B R^{-1} B^{T}\right]^{-1}$ :

$$
\begin{aligned}
& P_{\infty} \Delta x_{k}= \\
& =Q \Delta x_{k}+\phi^{T} P_{\infty}\left[I+B R^{-1} B^{T} P_{\infty}\right]^{-1} \phi \Delta x_{k} \\
& \forall \Delta x_{k} \Rightarrow D A R E \\
& -S_{1} \Delta r_{k}=-Q H \Delta r_{k} \\
& S_{2} \Delta r_{k+1}=-\phi^{T} M_{2} S_{1} \Delta r_{k+1} \\
& 0=\phi^{T} M_{2} S_{2} \Delta r_{k+2}
\end{aligned}
$$

Here, the first equation is the well known steady state Discrete Algebraic Riccati Equation (DARE) which means that it is satisfied in all time steps if $P \infty$ is calculated accordingly. The last three equations are automatically satisfied in case of constant references $\left(\Delta r_{k}=0 \forall k\right)$. However, for timevarying references they can not be satisfied only by using linear extrapolation of the reference signal. By using linear extrapolation, a different set of equations results:

$$
\begin{aligned}
& \Delta r_{k+2}=2 \Delta r_{k+1}-\Delta r_{k} \\
& -S_{1} \Delta r_{k}=-Q H \Delta r_{k}-\phi^{T} M_{2} S_{2} \Delta r_{k} \\
& S_{2} \Delta r_{k+1}=-\phi^{T} M_{2} S_{1} \Delta r_{k+1}+2 \phi^{T} M_{2} S_{2} \Delta r_{k+1}
\end{aligned}
$$

(7) is a system of equations for $S_{1}$ and $S_{2}$ which has a closed form solution:

$$
\begin{aligned}
& {\left[\begin{array}{cc}
I & -\phi^{T} M_{2} \\
\phi^{T} M_{2} & I-2 \phi^{T} M_{2}
\end{array}\right]\left[\begin{array}{l}
S_{1} \\
S_{2}
\end{array}\right]=\left[\begin{array}{c}
Q H \\
0
\end{array}\right] \Rightarrow\left[\begin{array}{l}
S_{1} \\
S_{2}
\end{array}\right]=} \\
& =\left[\begin{array}{cc}
I-\phi^{T} M_{2}\left(\left(I-\phi^{T} M_{2}\right)^{2}\right)^{-1} \phi^{T} M_{2} & (\ldots) \\
-\left(\left(I-\phi^{T} M_{2}\right)^{2}\right)^{-1} & \phi^{T} M_{2}
\end{array}\right]\left[\begin{array}{c}
Q H \\
0
\end{array}\right]
\end{aligned}
$$

The resulting control input consists of a state feedback and a reference feedforward part.

$$
\begin{aligned}
& \Delta u_{k}=-K_{x 2} \Delta x_{k}+\bar{K}_{S_{1}} \Delta r_{k+1}+K_{S_{2}} \Delta r_{k} \text { where } \\
& K_{x 2}=-R^{-1} B^{T} P_{\infty}\left[I+B R^{-1} B^{T} P_{\infty}\right]^{-1} \phi \\
& K_{S_{2}}=R^{-1} B^{T} M_{2} S_{2} \bar{K}_{S_{1}}=R^{-1} B^{T} M_{2} S_{1}-2 K_{S_{2}}
\end{aligned}
$$

From step 4. the resulting control input is the following (considering the estimated state $\hat{x}_{k}$ instead of the real $x_{k}$, and substituting $r_{k+1}$ in place of $r_{\infty}$ ):

$$
\begin{aligned}
& \tilde{u}_{k}=-K_{x} \hat{x}_{k}+\left(\bar{K}_{S_{1}}+K_{r_{\infty}}\right) r_{k+1}+K_{S_{2}} r_{k} \\
& \text { where }: \quad K_{x}=K_{x 1}+K_{x 2} \quad K_{r_{\infty}}= \\
& =K_{S_{2}}-K_{S_{1}}+\left(K_{x 2}(I-\phi)^{-1} B+I\right) \operatorname{pinv}(F)
\end{aligned}
$$

The properties of this new control solution has been derived and proven (the proofs are omitted due to the limited space) :

1) It satisfies the separation principle both for constant and time-varying references.

2) It does not require anti-windup compensation because of memoryless control.

3) It guarantees asymptotic stability, zero steady-state tracking error, finite LQ functional value (on infinite horizon!) and so, LQ optimality for constant references. 
4) It is LQ sub-optimal, BIBO and $l_{1} / l_{2}$ stable for timevarying references.

5) It guarantees finite tracking error in all time steps for ramp type references.

After deriving the control solution its tuning and testing was done using the lateral dynamical model of a small UAV (see [9]).

\section{AirCRAfT LATERAL DYNAMICAL MODEL}

This section first describes the used simulation model of the aircraft, then introduces the model used in control design and explains the steps of tuning and testing.

\section{A. The aircraft simulation model}

The lateral-directional aircraft model used in the article was derived from the model developed in [9]. Besides the linear aircraft dynamics, the model contains actuator dynamics and time delay (see Figure 1). $u, u_{0}, u_{1}$ are the input vectors including $\delta_{a}$ aileron and $\delta_{r}$ rudder deflections. $x$ is the state vector including $p$ rollrate, $r$ yaw-rate and $\phi$ roll angle. The tracking output $y^{r}$ will be defined later.

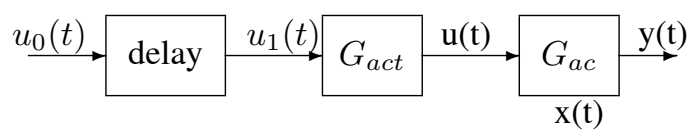

Fig. 1. The simulation model block diagram

The CT linear dynamic equation of the system $\left(G_{a c}\right)$ is:

$$
\underbrace{\left[\begin{array}{c}
\dot{p} \\
\dot{r} \\
\dot{\phi}
\end{array}\right]}_{\dot{x}}=\underbrace{\left[\begin{array}{ccc}
L_{p} & L_{r} & 0 \\
N_{p} & N_{r} & 0 \\
1 & 0 & 0
\end{array}\right]}_{A} \underbrace{\left[\begin{array}{c}
p \\
r \\
\phi
\end{array}\right]}_{x}+\underbrace{\left[\begin{array}{cc}
L_{\delta_{a}} & L_{\delta_{r}} \\
N_{\delta_{a}} & N_{\delta_{r}} \\
0 & 0
\end{array}\right]}_{B} \underbrace{\left[\begin{array}{c}
\delta_{a} \\
\delta_{r}
\end{array}\right]}_{u}
$$

The coefficients (aircraft stability and control derivatives) in $A$ and $B$ were obtained in [9] using system identification techniques. Three different model parameter sets resulted from three flight measurements. The parameters are summarized in Table I.

TABLE I

AIRCRAFT PARAMETERS

\begin{tabular}{|c|c|c|c|c|c|c|c|c|}
\hline Param. & $L_{p}$ & $L_{r}$ & $N_{p}$ & $N_{r}$ & $L_{\delta_{a}}$ & $L_{\delta_{r}}$ & $N_{\delta_{a}}$ & $N_{\delta_{r}}$ \\
\hline MOD1 & -12 & 12.7 & 0.294 & -8.48 & 58.1 & 13.6 & -6.58 & -17.5 \\
\hline MOD2 & -12.8 & 14.4 & -0.448 & -6.08 & 61.4 & 12.4 & -3.67 & -15 \\
\hline MOD3 & -11.1 & 8.62 & 0.687 & -4.62 & 43.3 & 8.99 & -4.76 & -11.9 \\
\hline
\end{tabular}

The considered actuator dynamics is (derived together with system identification):

$$
G_{a c t}=\frac{631.6}{s^{2}+35.2 s+631.6}
$$

The time delay in the controlled aircraft system is approximately $0.08 \mathrm{~s}$ published in [9] and verified by the authors in hardware in the loop (HIL) simulation. But tuning the controller for this delay gave unsatisfactory results in real flight tests so, the real delay should be larger. Examination of real flight data shown that the delay can be about $0.2 \mathrm{~s}$ so, this value was used finally. In the simulation model this was implemented as an integer delay.

\section{B. The model used in control design}

In the control design, a simplified model was used neglecting actuator dynamics and using the Padé approximation of delay (0.2s) (see Figure 2). An additional washout filter was inserted to select the high frequency component of yawrate.

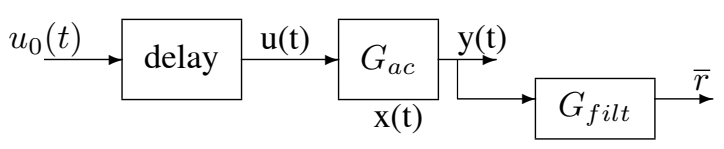

Fig. 2. The controlled model block diagram

For the Padé approximation of delay both first and second order functions were tested. The step response of the second order one is better, because it does not start from negative value so, finally it was selected (see Figure 3):

$$
\begin{aligned}
& G_{\text {delay }}=\frac{0.004 s^{2}-0.1 s+1}{0.004 s^{2}+0.1 s+1} \\
& \dot{x}^{d}=A_{d} x^{d}+B_{d} u \\
& \bar{u}=C_{d} x^{d}+D_{d} u
\end{aligned}
$$

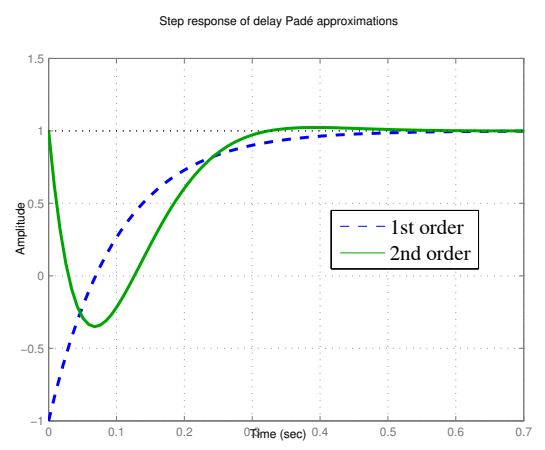

Fig. 3. Padé step responses

The applied washout filter was $G_{f i l t}=\frac{s}{s+15}$ (from [9]). Its equivalent state space representation is:

$$
\dot{x}^{F}=A_{F} x^{F}+B_{F} r, \quad \bar{r}=C_{F} x^{F}+D_{F} r
$$

Here $x^{F}$ is filter state, while $\bar{r}$ is the filtered yaw-rate. The augmented CT controlled system can be constructed from (11), (12) and (13) as follows: 


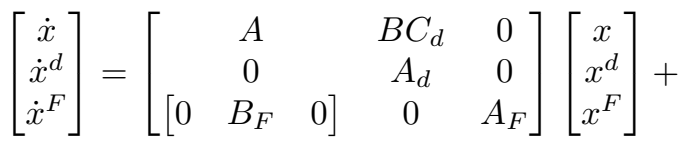

$$
\begin{aligned}
& +\left[\begin{array}{c}
B D_{d} \\
B_{d} \\
0
\end{array}\right] u \\
& y^{r}=\left[\begin{array}{l}
\phi \\
\bar{r}
\end{array}\right]=\left[\begin{array}{ccccc}
0 & 0 & 1 & 0 & 0 \\
0 & D_{F} & 0 & 0 & C_{F}
\end{array}\right]\left[\begin{array}{c}
p \\
r \\
\phi \\
x^{d} \\
x^{F}
\end{array}\right]
\end{aligned}
$$

(14) shows that the tracking outputs are aircraft roll angle and filtered yawrate.

\section{Controller tuning and testing}

The LQ optimal, infinite horizon output tracking controller derived in Section II was tuned to track roll angle reference commands and to hold the high frequency yawrate at zero on the system model (14). At first, the discrete time equivalent of the model was calculated for all three parameter sets (see Table I). Second, an averaged model was constructed from model 1 and 2. The poles of this discrete time augmented system model were:

$$
\begin{aligned}
p_{0}= & {\left[\begin{array}{llll}
1 & 0.5488 & 0.6145 & 0.7412
\end{array}\right] \& } \\
& {\left[\begin{array}{lll}
0.5616 \pm 0.2291 i & 0.5616 \pm 0.2291 i
\end{array}\right] }
\end{aligned}
$$

The prescribed stable and non-oscillating poles in step 1 were:

$$
\begin{aligned}
p= & {\left[\begin{array}{llll}
0.98 & 0.5488 & 0.6145 & 0.7412
\end{array}\right] \& } \\
& {\left[\begin{array}{llll}
0.5616 & 0.5616 & 0.6 & 0.6
\end{array}\right] }
\end{aligned}
$$

The weighting matrices were:

$$
\begin{gathered}
Q_{1}=<100,0,0,0,0,0,0,0> \\
Q_{2}=<2000,2>R=<5000,50000>
\end{gathered}
$$

Here $<>$ symbolizes a diagonal matrix. This shows that the roll rate $\left(Q_{1}(1,1)\right)$, the roll angle tracking error, the filtered yawrate and the control inputs were weighted. The resulting closed loop poles are:

$$
\begin{aligned}
& p_{c}=\left[\begin{array}{llll}
0.1856 & 0.536 & 0.5874 \pm 0.0158 i & 0.7279
\end{array}\right] \& \\
& {[0.7304 \pm 0.2567 i \quad 0.8432]}
\end{aligned}
$$

After tuning, the algorithm was tested in different simulations and in real flight. The test cases were the following:

1) Matlab test (ML) using the three different linear lateral dynamical model with delay $(0.2 \mathrm{~s})$ and actuator dynamics, implementing the control in Simulink blocks. The testing on three different models gives some proof of robustness.

2) Software in the loop (SIL) simulation using the full nonlinear aircraft model with delay $(0.2 \mathrm{~s})$ and actuator dynamics, implementing the control in $\mathrm{C}$ code.
3) Hardware in the loop (HIL) simulation using the full nonlinear aircraft model with delay $(0.2 \mathrm{~s})$ and actuator dynamics, implementing the control on the microcontroller.

4) Real flight (RF) test using the same microcontroller as in HIL.

The test results are summarized in the next section.

\section{TEST RESUlTS}

The ML test results are published in Figure 4. The LQ optimal controller was tested on all three linear models, while the baseline one only on the first model. The figure shows that the LQ optimal tracking results are a bit better. The settling times are summarized in Table II, some normalized signal 2norms $\left(\overline{\|x\|} \sqrt{\sum_{i=1}^{n} x_{i}^{2}} / n\right)$ are summarized in Table III.
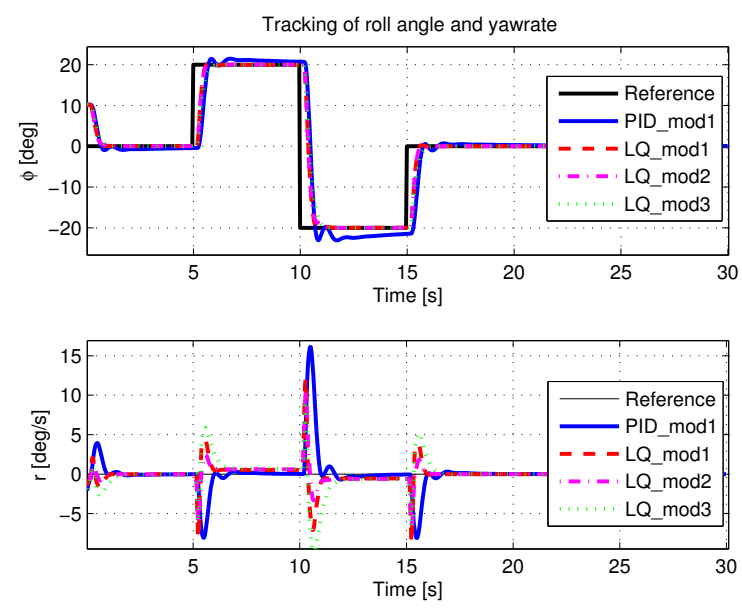

Fig. 4. ML simulation results

TABLE II

ML SETTLING TIMES

\begin{tabular}{|c|c|c|c|c|}
\hline Time & PID & $\mathrm{LQ} / 1$ & $\mathrm{LQ} / 2$ & $\mathrm{LQ} / 3$ \\
\hline$T_{s 1}[\mathrm{~s}]$ & 0.68 & 0.56 & 0.56 & 0.8 \\
\hline$T_{s 2}[\mathrm{~s}]$ & 0.68 & 0.68 & 0.92 & 0.96 \\
\hline
\end{tabular}

TABLE III

ML SIGNAL 2-NORMS

\begin{tabular}{|c|c|c|c|c|c|}
\hline Ctrl & $\phi_{\text {err }}$ & $\mathrm{p}$ & $r_{\text {err }}$ & $\delta_{a}$ & $\delta_{r}$ \\
\hline $\mathrm{PID}$ & 0.2271 & 0.5279 & 0.0768 & 0.0593 & 0.002 \\
\hline $\mathrm{LQ} / 1$ & 0.1935 & 0.528 & 0.0404 & 0.0621 & 0.02 \\
\hline $\mathrm{LQ} / 2$ & 0.1929 & 0.5259 & 0.0379 & 0.0619 & 0.0199 \\
\hline $\mathrm{LQ} / 3$ & 0.2034 & 0.4488 & 0.0638 & 0.063 & 0.0206 \\
\hline
\end{tabular}

Considering the settling times, the LQ controller is not better, but considering the 2-norms it is better in roll angle tracking $\left(\phi_{e r r}\right)$ and in yaw damping $\left(r_{\text {err }}\right)$. Of course with the cost of larger control input energy especially for $\delta_{r}$.

The SIL test results are published in Figures 5, 6. Here, the azimuth angle of aircraft was also calculated and examined. The motor reaction torque was considered in the nonlinear 
simulation and this caused problems with the LQ optimal tracking. The figure shows that it tracked the roll angle reference only with a constant deviation because lack of integral term in the control, but with much smaller overshoots then the baseline solution. The azimuth angles are also a bit worse, but yaw damping is better.
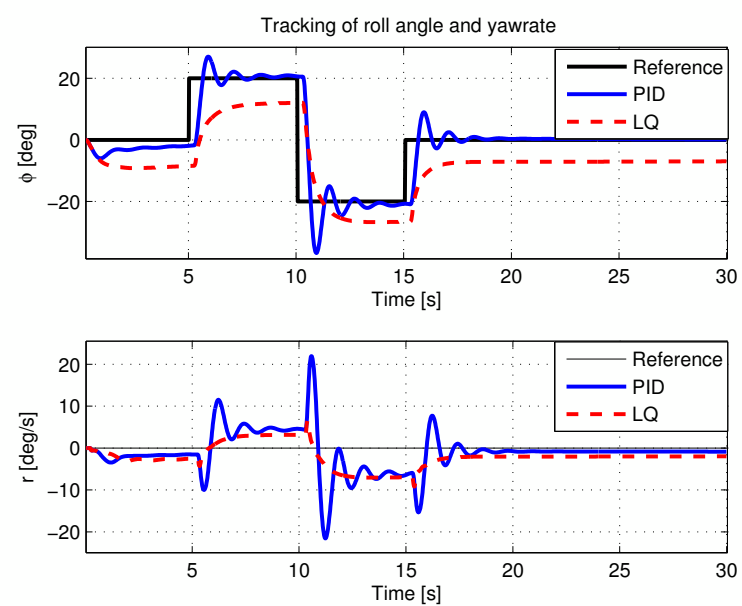

Fig. 5. SIL simulation results (roll angle and yawrate)

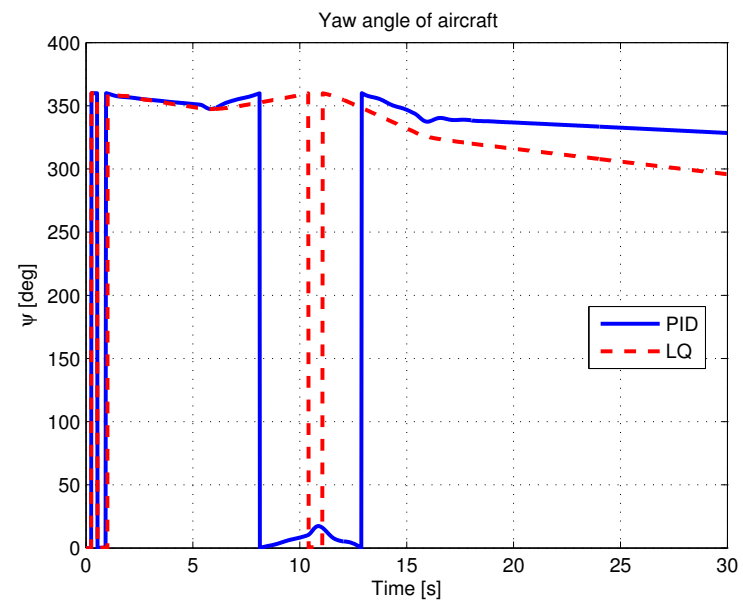

Fig. 6. SIL simulation results (azimuth angle)

TABLE IV

SIL SIGNAL 2-NORMS

\begin{tabular}{|c|c|c|c|c|c|}
\hline Ctrl & $\phi_{e r r}$ & $\mathrm{p}$ & $r_{\text {err }}$ & $\delta_{a}$ & $\delta_{r}$ \\
\hline PID & 0.1672 & 0.5157 & 0.1295 & 0.1228 & 0.0031 \\
\hline LQ & 0.2522 & 0.2404 & 0.0881 & 0.0685 & 0.0237 \\
\hline
\end{tabular}

The settling times were 1.36s and 1.32s for the PID and they can not be measured for the LQ because the roll angle is out of the $\pm 95 \%$ range. Signal 2-norms are summarized in Table IV. The Table shows that the LQ method gives smaller roll rate activity (smaller 2-norm) and less aileron control activity also.

The HIL test results are published in Figures 7, 8. Normalized signal 2-norms are summarized in Table $\mathrm{V}$. The results are similar to the SIL case. The yaw damping of LQ is better, but with larger control energy. The constant deviation of roll angle also occurs.
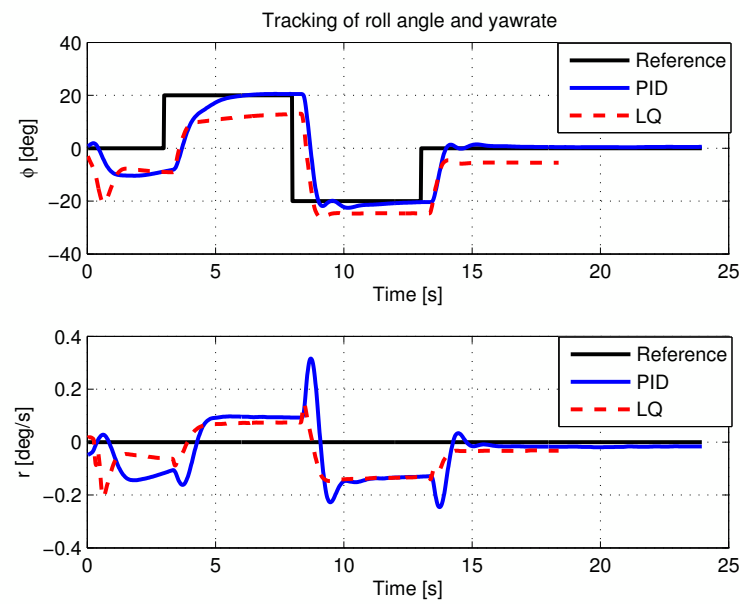

Fig. 7. HIL simulation results (roll angle and yawrate)

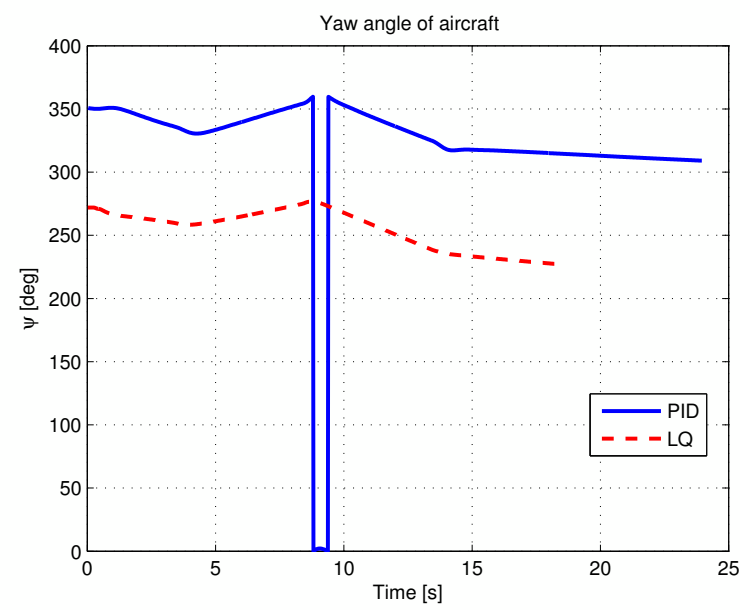

Fig. 8. HIL simulation results (azimuth angle)

TABLE $\mathrm{V}$

HIL SIGNAL 2-NORMS

\begin{tabular}{|c|c|c|c|c|c|}
\hline Ctrl & $\phi_{e r r}$ & $\mathrm{p}$ & $r_{\text {err }}$ & $\delta_{a}$ & $\delta_{r}$ \\
\hline PID & 0.2741 & 0.4124 & 0.1672 & 0.0707 & 0.0042 \\
\hline LQ & 0.372 & 0.5482 & 0.1658 & 0.0857 & 0.0337 \\
\hline
\end{tabular}

The last case is real flight testing. Results are published in Figures 9, 10. Normalized signal 2-norms are summarized in Table VI.

TABLE VI

FLIGHT TEST SIGNAL 2-NORMS

\begin{tabular}{|c|c|c|c|c|c|}
\hline Ctrl & $\phi_{\text {err }}$ & $\mathrm{p}$ & $r_{\text {err }}$ & $\delta_{a}$ & $\delta_{r}$ \\
\hline PID & 0.283 & 0.9 & 0.2894 & 0.0926 & 0.0136 \\
\hline LQ & 0.1775 & 0.9 & 0.2367 & 0.071 & 0.0213 \\
\hline
\end{tabular}



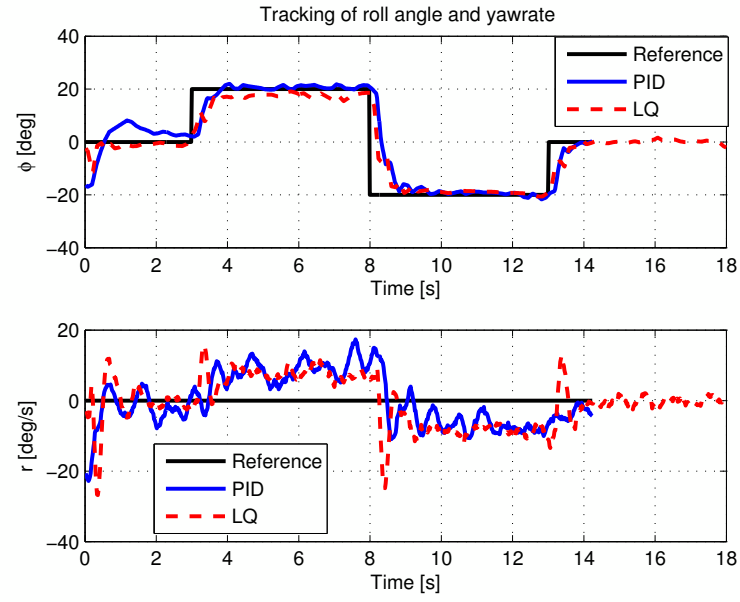

Fig. 9. Flight test results (roll angle and yawrate)

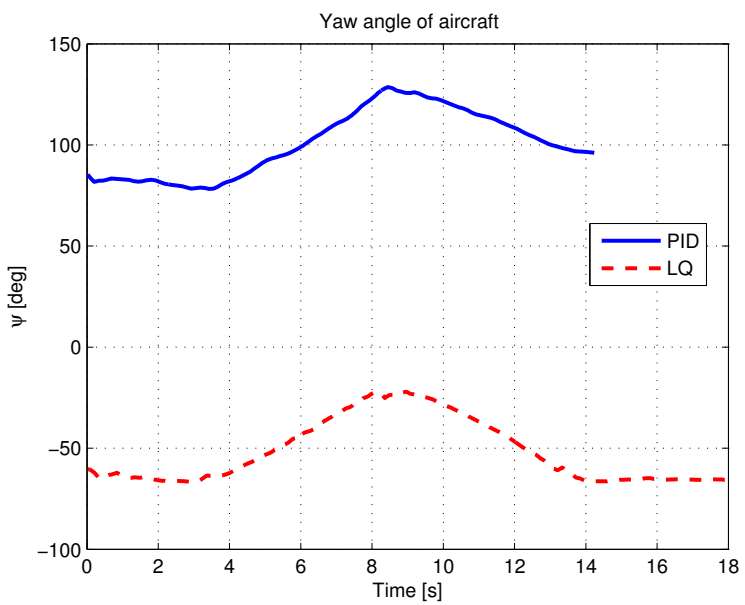

Fig. 10. Flight test results (azimuth angle)

The results are very different from expected (from SIL and HIL). Both yaw damping and roll angle tracking are much better with LQ then with the baseline controller (see Figures and signal 2-norms). The aileron control activities are smaller, the rudder activities are larger considering the 2-norms.

\section{CONCLUSION}

This paper described a LQ optimal, infinite horizon output tracking solution from problem formulation to real flight tests.

It started with literature review, then rigorously derived the sub-optimal (for time-varying references) solution together with the optimal one (for constant references). It stated all the properties of the derived solution.

Then it introduced the lateral-directional aircraft model on which the algorithm was tuned and tested (see [9]). The LQ optimal algorithm was compared by a baseline PID control (see again [9]). After publishing the steps of tuning and testing, the test results were plotted.

At first, the Matlab simulation results for the three different linear models were evaluated. Then SIL and HIL simulation results were plotted. Finally, real flight test results were summarized.
As a summary, it can be stated that the results are satisfactory especially in real flight. The LQ control is a bit better then the baseline controller. Its main advantage is the simple structure, the smaller overshoots and that it does not need anti-windup compensation. The largest problem of this control method is the sensitivity to constant disturbances such as motor torque - which cause constant tracking errors. This problem can be (and was) solved by deriving a minimax tracking control solution (see [10]).

\section{ACKNOWLEDGMENT}

The authors gratefully acknowledge to the support by the Hungarian National Scientific Research Fund (OTKA CNK 78168), by the TRUCKDAS project (TECH_08-A2/2-20080088) and by the Control Engineering Research Group of HAS at BUTE.

\section{REFERENCES}

[1] J. Burl, Linear Optimal Control, $\mathcal{H}_{2}$ and $\mathcal{H}_{\infty}$ methods. Addision Wesley Longman Inc., 1999.

[2] M. Athans and P. Falb, Optimal Control, An Introduction to the Theory and its Applications. McGraw-Hill Book Company, 1966.

[3] B. Anderson and J. Moore, Optimal Control, Linear Quadratic Methods. Prentice Hall, Englewood Cliffs, NJ, 1989.

[4] F. L. Lewis, Optimal Control. John Wiley \& Sons, 1986.

[5] J. L. Willems and I. Mareels, "A Rigorous Solution of the Infinite Time Interval LQ Problem with Constant State Tracking," Systems and Control Letters, vol. 52, pp. 289-296, 2004.

[6] E. Barbieri and R. Alba-Flores, "On the Infinite-horizon LQ Tracker," Systems and Control Letters, vol. 40, pp. 77-82, 2000.

[7] P. Bauer, B. Kulcsr, and J. Bokor, "An Exact Solution for the Infinite Horizon LQ Optimal Output Tracking Problem," in Proc. of 2008 IEEE Multi-Conference on Systems and Control, San Antonio, Texas, USA, 2008, pp. 822-827.

[8] P. Bauer, "The properties of an infinite horizon LQ optimal tracker with time varying references," in Proc. of Vehicle System Dynamics Identification and Anomalies (VSDIA) 2008, Budapest, Hungary, 2008, pp. 645-652.

[9] Y. C. Paw, "Synthesis and Validation of Flight Control for UAV," Ph.D. dissertation, University of Minnesota, 2009.

[10] P. Bauer and J. Bokor, "Tuning and testing of a minimax tracking controller for aircraft dynamics," in Proc. of IEEE 19th Mediterranean Conference on Control and Automation, Corfu, Greece, 2011, pp. 259264. 